\title{
Heat Transfer Coefficient at Cast-Mold Interface During Centrifugal Casting: Calculation of Air Gap
}

\author{
JAN BOHACEK, ABDELLAH KHARICHA, ANDREAS LUDWIG, MENGHUAI WU, \\ and EBRAHIM KARIMI-SIBAKI
}

During centrifugal casting, the thermal resistance at the cast-mold interface represents a main blockage mechanism for heat transfer. In addition to the refractory coating, an air gap begins to form due to the shrinkage of the casting and the mold expansion, under the continuous influence of strong centrifugal forces. Here, the heat transfer coefficient at the cast-mold interface $h$ has been determined from calculations of the air gap thickness $d_{a}$ based on a plane stress model taking into account thermoelastic stresses, centrifugal forces, plastic deformations, and a temperature-dependent Young's modulus. The numerical approach proposed here is rather novel and tries to offer an alternative to the empirical formulas usually used in numerical simulations for a description of a time-dependent heat transfer coefficient $h$. Several numerical tests were performed for different coating thicknesses $d_{\mathrm{C}}$, rotation rates $\Omega$, and temperatures of solidus $T_{\mathrm{sol}}$. Results demonstrated that the scenario at the interface is unique for each set of parameters, hindering the possibility of employing empirical formulas without a preceding experiment being performed. Initial values of $h$ are simply equivalent to the ratio of the coating thermal conductivity and its thickness $\left(\sim 1000 \mathrm{Wm}^{-2} \mathrm{~K}^{-1}\right)$. Later, when the air gap is formed, $h$ drops exponentially to values at least one order of magnitude smaller $\left(\sim 100 \mathrm{Wm}^{-2} \mathrm{~K}^{-1}\right)$.

https://doi.org/10.1007/s11663-018-1220-0

(C) The Author(s) 2018. This article is an open access publication

\section{INTRODUCTION}

HORIZONTAL centrifugal casting is an important industrial process used especially for the production of high-quality seamless tubes and outer shells of work rolls. In this process, the effect of centrifuging is twofold. First, it is the fictitious centrifugal force making the production of axisymmetric hollow castings even possible by pushing the molten metal against the inner wall of the cylindrical mold. Second, the interaction between inertial forces and the vector of the gravitational acceleration induces the so-called pumping effect, responsible for thorough mixing, ${ }^{[1]}$ the growth of fine equiaxed grains, and superior mechanical properties of the cast. ${ }^{[2,3]}$

As with many other industrial processes, horizontal centrifugal casting has been studied with increased

JAN BOHACEK, ABDELLAH KHARICHA, and ANDREAS LUDWIG are with the Chair of Simulation and Modeling Metallurgical Processes, Metallurgy Department, Montanuniversitaet Leoben, Franz-Josef-Str. 18/III, 8700 Leoben, Austria. Contact e-mail: jan.bohacek@unileoben.ac.at MENGHUAI WU and EBRAHIM KARIMI-SIBAKI are with the Christian Doppler Laboratory for

"Advanced Process Simulation of Solidification and Melting,", Franz-Josef-Str. 18/III, 8700 Leoben, Austria.

Manuscript submitted October 26, 2016.

Article published online March 12, 2018. attention, with the help of various numerical techniques, in order to gain a better understanding of the process and underlying physical phenomena. While some of the numerical studies concentrate more on simulating flow dynamics, such as the mold filling, waves propagating over the free surface, and complex buoyant flow patterns inside the molten metal, ${ }^{[4-11]}$ others focus more on heat transfer and solidification, often assuming coupling with simple segregation models. ${ }^{[12-14]}$ The latter is naturally more frequent within the centrifugal casting community. Solidification is usually modeled by means of applying the enthalpy method with appropriate rules for a liquid fraction evolution in the mushy zone. In order to construct useful and realistic heat transfer models, precise and accurate material properties and boundary conditions are necessary. Heat transfer coefficients are usually imposed at boundaries, generally being determined from empirical formulas for the Nusselt number. Materials properties are generally temperature dependent and must be specified for all zones, i.e., the casting, the mold, and the coating. The thickness of the coating, a kind of a refractory material, such as $\mathrm{ZrO}_{2}$, is usually small $(\sim 1 \mathrm{~mm})$; therefore, in numerical models, it is often simplified by an assumption of the thin-wall (zero-capacity) model. The coating is applied on the inner surface of the mold in order to insulate the mold from high temperatures and also to control to a certain extent the solidification rate. The 
general consensus is that it tends to stick firmly to the mold surface. A time-dependent scenario at the contact between the casting and the coating attached to the mold surface is perhaps one of the weakest points of all currently available heat transfer models.

Only during the first seconds of the casting, the molten metal is in perfect contact with the coating, as pointed out in Reference 15. Immediately after that, a so-called microscopic air gap is formed, whose properties such as thickness and temporal growth are strongly influenced by a surface roughness of the mold and the coating eventually. Earlier studies ${ }^{[16]}$ show that the importance of the surface roughness has been for many years underestimated. Furthermore, in the literature (e.g., Reference 17), there are interesting numerical works available, taking into account the surface roughness and trying to evaluate the effective thickness of the microscopic air gap by using simple geometrical operations. As time proceeds, the first layer of the solid has enough strength to withstand the metallostatic pressure, whereby the air gap thickness gradually grows and the microscopically thin contact is permanently lost. Such an air gap is often referred to as the macroscopic air gap. Please note that while the cast-mold contact has been extensively studied in static castings and a large body of experimental evidence of microscopic and macroscopic air gap behavior has been presented, it is not yet clear whether at least qualitatively the same observations would apply to the centrifugal casting. Unlike static casting, extreme centrifugal forces are exerted on the liquid metal being cast, which strive to delay the subsequent air gap formation. We deduce that the high centrifugal pressure may be able to significantly reduce the microscopic air gap. Furthermore, we also believe-and this has been proven in this article - that once the macroscopic air gap is formed, the centrifugal force has a negligible impact on its growth.

In order to cope with time-dependent thermal resistance induced by the formation of an air gap, various approaches have been adopted in earlier numerical studies of centrifugal casting. Naturally, a simplest approach would be an assumption of a perfect contact formed throughout the entire casting, which was used, e.g., by $\mathrm{Xu}$ et al., ${ }^{[18]} \mathrm{Gao}$ and Wang, ${ }^{[19]}$ and Cook et al. ${ }^{[20]}$ Bohacek et al. ${ }^{[21]}$ pointed out the importance of an air gap in their findings and conclusions, yet in the numerical model, it was not taken into account. Humphreys et al. ${ }^{[22]}$ calculated the heat transfer at the interface by employing a "virtual wall" technique with cumulative thermal resistances; however, they did not provide parameters to calculate them. Chang et al. ${ }^{[23]}$ used arbitrary values of heat transfer coefficients at the interface, constant during the casting and increasing for higher rotation rates. Other researchers, such as Kang et $a .^{[24]}$ and Kang and Rohatgi, ${ }^{[25]}$ have also used time-independent heat transfer coefficients. Ebisu ${ }^{[26]}$ and Kamlesh $^{[27]}$ assumed an exponential decay of the radiative heat flux through the interface as follows:

$$
q=q_{0} e^{-\beta s(t)},
$$

where $q_{0}, \beta$, and $s(t)$ are the initial heat flux through the interface, a damping coefficient, and the current solidified thickness, respectively. A similar approach was adopted by Lajoye and Suery ${ }^{[28]}$ and was later widely used by other authors such as Raju and Mehrotra, ${ }^{[29]}$ Drenchev et al., ${ }^{[30]}$ Panda et al. ${ }^{[31]}$ Instead of the heat flux, a time-dependent heat transfer coefficient $h$ was considered at the interface and defined by the following formula:

$$
h=h_{0}\left(\frac{h_{\mathrm{f}}}{h_{0}}\right)^{s(t) / d},
$$

where $h_{0}, h_{\mathrm{f}}$, and $d$ are the initial and the final heat transfer coefficient and the casting thickness, respectively. Naturally, Eqs. [1] and [2] are not equivalent. However, it is worth noting that when the heat flux $q$ was replaced with the heat transfer coefficient $h$, Eqs. [1] and [2] would become identical provided that the damping coefficient was defined as

$$
\beta=-\frac{1}{d} \log \left(\frac{h_{\mathrm{f}}}{h_{0}}\right) \text {. }
$$

Recently, Nastac ${ }^{[32]}$ applied a different approach based on calculating an equivalent convective heat transfer coefficient $h$ to simulate the effect of the coating and the air gap, which can be written as

$$
h=\frac{h_{\mathrm{a}} k_{\mathrm{C}}}{k_{\mathrm{C}}+d_{\mathrm{C}} h_{\mathrm{a}}},
$$

where $k_{\mathrm{C}}, d_{\mathrm{C}}$, and $h_{\mathrm{a}}$ represent thermal conductivity and thickness of the coating and the heat transfer coefficient between the casting and the coating, which is defined as follows:

$$
h_{\mathrm{a}}=h_{0}+\left(h_{\mathrm{f}}-h_{0}\right)\left\{1-\left[\min \left(1, \frac{t_{0}}{t}\right)\right]^{\gamma}\right\},
$$

where $t_{0}, t$, and $\gamma$ stand for the time to initiation of solidification, the current time, and a constant exponent. Table I summarizes the values of $h$ adopted by the aforementioned authors. Obviously, all of the aforementioned approaches contain at least one unknown parameter, which needs to be adjusted, e.g., by means of an experiment. Although especially the choice of the function given by Eq. [2] appears to be a reasonable solution, a careful fine-tuning of $h_{\mathrm{f}}$ is required in order to reflect, or at least approximate, real-life conditions. According to Vacca et al., ${ }^{[33]}$ who performed a valuable experimental study of the heat transfer coefficient at the interface involving the inverse task, values of the heat transfer coefficient adopted in centrifugal casting simulations are unreliable and usually arbitrary. A similar approach combining a simulation and experiment was employed by Susac et al. ${ }^{[34]}$ and Sahin et al. ${ }^{[35]}$ The inverse task is, however, in general, computationally very intensive. Moreover, time-dependent experimental data are necessary at least at one point, located close to the castmold interface. While the inverse task cannot be practically applied in a typical centrifugal casting simulation, it is an excellent tool for validating other numerical models or determining constants in 
empirical models. In Reference 36, the inverse task, the inverse heat conduction problem (IHCP), was solved by a popular nonlinear estimation technique, originally developed by Beck. ${ }^{[37]}$ The casting material, A356 Al alloy, was cast into a carbon steel mold. The IHCP proved that the heat transfer to the mold can be significantly improved by applying the pressure load during solidification in terms of restoring a contact between the mold and the casting.

In Reference 38, the research concerns the simulation of trip continuous casting. An engineering approach was employed to approximate the thermal resistance at the strip-mold interface from the heat transferred to the cooling water. The water flow rate and temperature were recorded at different positions along the length of the strip for this purpose. At selected points in the liquid pool, the calculated cooling curves agreed strongly with those obtained from Inconel (American Special Metals, Corp., Miami, FL) sheathed thermocouples.

In addition, a direct measurement of the macroscopic air gap can be performed by using linear variable differential transformers (LVDTs). However, this technique during the centrifugal casting is limited to static castings due to high rotations of the mold during centrifugal casting. For example, in Reference 39, the heat transfer coefficient at the cast-mold interface of a static casting was determined from the inverse task. The air gap thickness was measured with the help of the LVDTs. Finally, a correlation was found, defining the heat transfer coefficient as a function of the air gap thickness. The effect of the surface roughness of the mold was analyzed. As expected, during formation of the microscopic air gap, findings showed that the smaller degree of roughness provides stronger contact with the mold and that the heat transfer coefficient is, therefore, higher. Consequently, the smaller the surface roughness of the mold, the earlier the macroscopic air gap occurs. On the other hand, the ultimate heat transfer coefficient, when solidification is nearly complete, is insignificantly influenced by the surface roughness.
As a numerical alternative of estimating the air gap thickness, one could suggest ignoring stresses built up in the casting and the mold and using the thermal expansion coefficient to calculate the shrinkage simply by assuming displacements, independent of direction, i.e., uniform rate of deformation of control volume. This technique was applied, e.g., by Taha et al., ${ }^{[40]}$ for a static casting. Accuracy and reliability are, however, doubtful due to the missing thermal stresses, which may significantly alter total displacements. In addition, unlike static castings, extreme forces in the centrifugal casting process act on the casting, leading to yielding of the material especially at early stages. In addition, for this reason, the aforementioned approach should be avoided. A strategy that incorporates a more complete and holistic model of physics was outlined by Kron, ${ }^{[41]}$ who suggested taking into account vacancies formed due to the thermal expansion of the mold and the material being cast, as well as elastic stresses acting as a consequence of thermally induced strains. Kron et al. ${ }^{[42]}$ developed the thermomechanical model, based on the plane stress model, assuming elastic materials. They showed that the model predicts accurately the casting scenario only for grain-refined alloys such as Al-4.5 pct Mg. When the solid grains are surrounded by liquid, the material becomes more ductile and consequently the microscopic air gap is suppressed. On the other hand, in the non-grain-refined case, the elastic thermomechanical model does not perform particularly effectively. However, one may wish to interpret these findings, the important message of Reference 42 could be formulated as follows: The peak value of the heat transfer coefficient is larger for the grain-refined alloy due to ductile suppression of the microscopic air gap, but since the formation of the macroscopic air gap starts earlier in this case, total solidification times are almost identical. Lagerstedt, a colleague of Kron, pointed out in the future work chapter of his doctoral thesis ${ }^{[43]}$ that including plasticity in the stress model probably should be the next step in developing an accurate shrinkage model.

Table I. Values of $\boldsymbol{h}$ Adopted by Different Authors

\begin{tabular}{|c|c|c|}
\hline Authors & Type of $h$ & Value $\left(\mathrm{W} \mathrm{m}^{-2} \mathrm{~K}^{-1}\right)$ \\
\hline $\mathrm{Xu}$ et $a ._{.}^{[18]}$ & - & perfect contact \\
\hline Gao and Wang ${ }^{[19]}$ & - & perfect contact \\
\hline Cook et al..$^{[20]}$ & - & perfect contact \\
\hline Bohacek et al. ${ }^{[21]}$ & - & perfect contact \\
\hline Humphreys et al. ${ }^{[22]}$ & - & not available \\
\hline Chang et al. ${ }^{[23]}$ & constant & 1000 to 2600 \\
\hline Kang et al., ${ }^{[24]}$ Kang and Rohatgi ${ }^{[25]}$ & constant & 1000 \\
\hline $\mathrm{Ebisu}^{[26]}$ & variable & not available \\
\hline Kamlesh $^{[27]}$ & variable & not available \\
\hline Lajoye and Suery ${ }^{[28]}$ & variable & 400 to $80,000(1 / 10)^{*}$ \\
\hline Raju and Mehrotra ${ }^{[29]}$ & variable & 100 to $10,000(1 / 10)$ \\
\hline Drenchev et al. ${ }^{[30]}$ & variable & 420 to $84,000(1 / 10)$ \\
\hline Panda et al. ${ }^{[31]}$ & variable & 50 to $5000(1 / 10)$ \\
\hline Nastac $^{[32]}$ & variable & 90 to $6000(3 / 100)$ \\
\hline Vacca et al. ${ }^{[33]}$ & variable (exp.) & 50 to $870(6 / 100)$ \\
\hline
\end{tabular}


In Reference 44, Schwerdtfeger et al. underlined the importance of the displacement reference. Classically, in stress theory, the displacement is the distance of a specified atom from the position it had assumed when the entire solid body was stress free. Such a situation, however, does not occur during solidification; therefore, they recommended defining the displacement as the distance of a specified atom from the position where it was at the moment of solidification. Consequently, one should work with stress rates and strain rates rather than with stresses and strains. In their study, plastic deformations were also considered and added to the elastic ones by assuming an empirical strain-hardening equation.

Nowadays, most of the commercial software available on the market, including MAGMASOFT (MAGMA in Aachen, North Rhine-Westphalia, Germany), PROCAST (ESI Group, Paris, France), and THERCAST (TRANSVALOR S.A., Mougins, France), offers modules for thermomechanical calculations, and often the user can choose from several elastic-plastic models. In Reference 45, in conclusion, Kron et al. stated that an accurate modeling of the air gap formation can only be realized through fully coupled thermomechanical models. They highlighted that the prediction of the air gap, done with the commercial codes, is not satisfactory, suggesting that the solidification shrinkage in the air gap vicinity should be relaxed by the liquid and, therefore, contribute more to a cavity formed in the top of the casting or to the porosity. In conclusion, the entire strain model needs to be defined more precisely. Difficulties associated with air gap modeling were summarized in Reference 46 as the following. High-temperature elastic constants are generally hard to obtain. Defining material properties of the mushy zone remains a challenging topic. Currently, a transition model is available described by the Percolation theory. Next, obtaining proper values of rheological parameters in the power law equation is difficult. Finally, other difficulties or discrepancies, common to all numerical models, are related to oversimplifying assumptions and numerical errors.

In the article by Nayak and Sundarraj, ${ }^{[47]}$ it was shown that while it is accurate enough to assume a constant value of the interface heat transfer coefficient during the entire casting into the sand mold, it is not the case with the metal mold. Furthermore, the rate of gap formation significantly affects the solidification process.

The cast-mold interface, namely, the coating and an air gap, represents a significant blockage for the heat transfer and solidification. The thermal resistance of the coating is often negligible compared to that of the air gap. Therefore, a thermal resistance of such an interface has to be carefully determined in order to allow reliable and trustworthy numerical simulations. Existing empirical formulas describing the heat transfer scenario at the interface should only be applied when validated against experimental data. The formation of an air gap depends on many factors such as material and mechanical properties of the casting and the mold, the coating properties, and the process parameters (initial temperatures, the pouring temperature, the casting geometry, and the rotation rate). Obviously, setting up a generalized and unique formula for the heat transfer coefficient at the interface a priori would be very hard, if not impossible. In the present article, we target developing a simple, computationally cheap, and robust algorithm for calculating the air gap at the cast-mold interface during the centrifugal casting, which could be used as an alternative to often doubtful empirical formulas. A schematic of the configuration at the interface is shown in Figure 1.

\section{NUMERICAL MODEL}

During the centrifugal casting of cylindrical parts, it is reasonable to assume that fields of variables and other properties are uniform in the tangential direction. In fact, also, axial variations will be often small and, therefore, could be neglected, too. This finding directly suggests using a plane stress model. Although different variations of plane stress models have been used in diverse industrial applications, such as autofrettage of gun barrels, strain-hardened pressure vessels, and multilayer seamless pipes, in the past, ${ }^{[48-51]}$ they have rarely been employed in air gap thickness calculations. Especially, concerning the centrifugal casting, to the best of our knowledge, not a single match was found in the literature survey.

Radial and tangential stresses, $\sigma_{\mathrm{r}}$ and $\sigma_{\mathrm{t}}$, are coupled through the equilibrium equation:

$$
\frac{\mathrm{d} \sigma_{\mathrm{r}}}{\mathrm{d} r}+\frac{\sigma_{\mathrm{r}}-\sigma_{\mathrm{t}}}{r}+\rho \Omega^{2} r=0,
$$

where $\rho, \Omega$, and $r$ are the density, the rotation rate, and the radial coordinate, respectively. When only elastic deformations are considered, stresses are coupled with strains via the Hooke's law with the thermoelastic term. Such a relationship takes the following form:

$$
\begin{aligned}
& \varepsilon_{\mathrm{t}}=\frac{1}{E}\left[\sigma_{\mathrm{t}}-v \sigma_{\mathrm{r}}\right]+\alpha T, \\
& \varepsilon_{\mathrm{r}}=\frac{1}{E}\left[\sigma_{\mathrm{r}}-v \sigma_{\mathrm{t}}\right]+\alpha T,
\end{aligned}
$$

where $v, E, \alpha$, and $T$ are Poisson's ratio, Young's modulus, the thermal expansion coefficient, and the temperature, respectively. Strains and total radial displacements are related through the following laws:

$$
\varepsilon_{\mathrm{t}}=\frac{u}{r}, \varepsilon_{\mathrm{r}}=\frac{\mathrm{d} u}{\mathrm{~d} r} .
$$

It would, however, be incorrect to only consider elastic strains. Since the casting in a semisolid state can easily yield under strong centrifugal forces, plastic deformations also must be taken into account. Then, total strains can be conveniently expressed as a sum of elastic and plastic strains as follows:

$$
\begin{aligned}
& \varepsilon_{\mathrm{t}}=\frac{1}{E}\left[\sigma_{\mathrm{t}}-v \sigma_{\mathrm{r}}\right]+\alpha T+\varepsilon_{\mathrm{t}}^{\mathrm{p}}, \\
& \varepsilon_{\mathrm{r}}=\frac{1}{E}\left[\sigma_{\mathrm{r}}-v \sigma_{\mathrm{t}}\right]+\alpha T+\varepsilon_{\mathrm{r}}^{\mathrm{p}},
\end{aligned}
$$

where $\varepsilon_{\mathrm{t}}^{\mathrm{p}}$ and $\varepsilon_{\mathrm{r}}^{\mathrm{p}}$ represent plastic strains in corresponding directions. In addition, it is also physically meaningful to consider a temperature-dependent Young's modulus. Substituting total strains in Eq. [9] with displacements from Eq. [8], and by combining the 


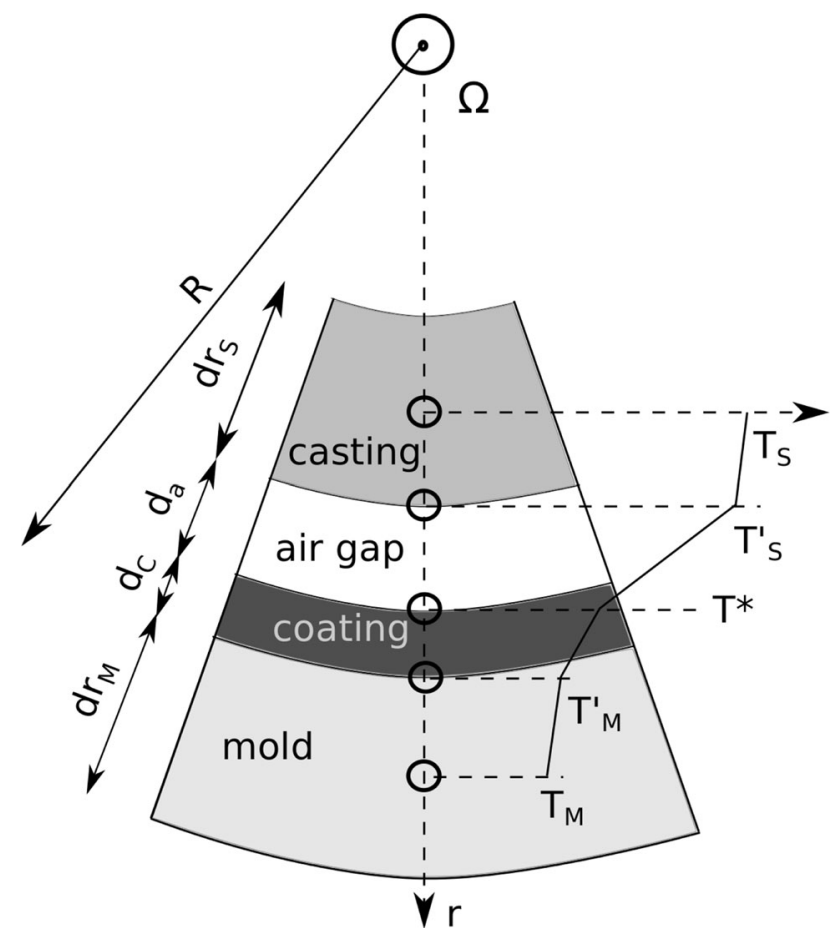

Fig. 1-Schematic of the configuration at the cast-mold interface. In the case of perfect contact, the air gap disappears.

resulting equations with Eq. [6], we can arrive at the following ordinary differential equation for the total displacement $u$ in the casting:

$$
\begin{aligned}
& \frac{\mathrm{d}^{2} u}{\mathrm{~d} r^{2}}+\frac{1}{r} \frac{\mathrm{d} u}{\mathrm{~d} r}\left(1+r \frac{1}{E} \frac{\mathrm{d} E}{\mathrm{~d} r}\right)-\frac{u}{r^{2}}\left(1-r v \frac{1}{E} \frac{\mathrm{d} E}{\mathrm{~d} r}\right) \\
& =\alpha(1+v)\left(\frac{\mathrm{d} T}{\mathrm{~d} r}+T \frac{1}{E} \frac{\mathrm{d} E}{\mathrm{~d} r}\right)-\rho r \Omega^{2}\left(1-v^{2}\right) \frac{1}{E} \\
& \quad+F\left(\varepsilon_{\mathrm{t}}^{\mathrm{p}}, \varepsilon_{\mathrm{r}}^{\mathrm{p}}\right) w i t h F=\frac{1}{E} \frac{\mathrm{d} E}{\mathrm{~d} r}\left(\varepsilon_{\mathrm{r}}^{\mathrm{p}}+v \varepsilon_{\mathrm{t}}^{\mathrm{p}}\right)+\frac{\mathrm{d} \varepsilon_{\mathrm{r}}^{\mathrm{p}}}{\mathrm{d} r}+v \frac{\mathrm{d} \varepsilon_{\mathrm{t}}^{\mathrm{p}}}{\mathrm{d} r} \\
& \quad+\frac{1-v}{r} \varepsilon_{\mathrm{r}}^{\mathrm{p}}-\frac{1-v}{r} \varepsilon_{\mathrm{t}}^{\mathrm{p}} .
\end{aligned}
$$

In the mold, Eq. [10] is considerably simplified because one assumes a constant Young's modulus and pure elastic deformations. The differential equation for the total displacement $u$ in the mold becomes

$$
\frac{\mathrm{d}^{2} u}{\mathrm{~d} r^{2}}+\frac{1}{r} \frac{\mathrm{d} u}{\mathrm{~d} r}-\frac{u}{r^{2}}=\alpha(1+v) \frac{\mathrm{d} T}{\mathrm{~d} r}-\rho r \Omega^{2}\left(1-v^{2}\right) \frac{1}{E} .
$$

Note that in Eqs. [10] and [11], subscripts $[\ldots]_{S}$ and $[\ldots]_{M}$ denoting the mold and the casting are omitted for the sake of brevity. Equations [10] and [11] can be solved provided that plastic strains $\varepsilon_{\mathrm{r}}^{\mathrm{p}}$ and $\varepsilon_{\mathrm{t}}^{\mathrm{p}}$ are known. In order to determine them, a universal stress-strain curve, usually assumed to be equivalent to the stress-strain curve obtained from the uniaxial loading test, must be known in advance. The universal stress-strain curve relates two scalar quantities: the effective stress $\bar{\sigma}$ and the effective plastic strain $\overline{\varepsilon^{\mathrm{p}}}$. Several models of the effective stress $\bar{\sigma}$ exist. Here, the von Mises stress,

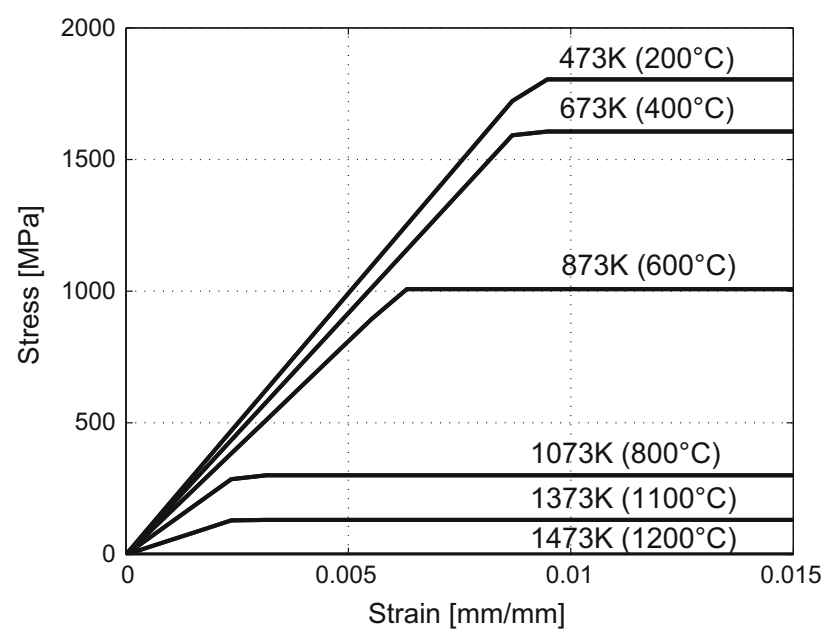

Fig. 2-Uniaxial stress-strain curves of elastic-perfectly plastic material used in the present study.

calculated by assuming a principal stress loading, was used in the following form:

$$
\bar{\sigma}=\frac{1}{\sqrt{2}} \sqrt{\left(\sigma_{\mathrm{r}}-\sigma_{\mathrm{t}}\right)^{2}+\sigma_{\mathrm{r}}^{2}+\sigma_{\mathrm{t}}^{2}} .
$$

The von Mises stress $\bar{\sigma}$ is coupled with the increment of the effective plastic strain $\overline{d \varepsilon^{\mathrm{p}}}$ by the Prandtl-Reuss (Levy-Mises) flow rule as follows:

$$
d \varepsilon_{\mathrm{r}}^{\mathrm{p}}=\frac{\overline{d \varepsilon^{\mathrm{p}}}}{\bar{\sigma}}\left(\sigma_{\mathrm{r}}-0.5 \sigma_{\mathrm{t}}\right)
$$

and

$$
d \varepsilon_{\mathrm{t}}^{\mathrm{p}}=\frac{\overline{d \varepsilon^{\mathrm{p}}}}{\bar{\sigma}}\left(\sigma_{\mathrm{t}}-0.5 \sigma_{\mathrm{r}}\right) .
$$

Knowing or assuming $\overline{d \varepsilon^{p}}$, the plastic strains $d \varepsilon_{r}^{p}$ and $d \varepsilon_{t}^{p}$ can be easily calculated and then used in Eq. [10] to extrapolate the total displacement $u$. In Eqs. [13] and [14], the increment of the effective plastic strain $\overline{d \varepsilon^{p}}$ is used and not the effective plastic strain $\overline{\varepsilon^{p}}$, which means that the plastic strain history (or the loading path) is very important. Correspondingly, a progressive load must be also applied in the simulation. Here, such loading is automatically realized by a time-dependent temperature field and a gradual progress of solidification.

In the present study, a temperature-dependent, perfectly elastic-plastic material is used, as shown in Figure 2. At a given temperature, the material deforms elastically until a certain threshold of the effective stress, known as the yield strength, is reached, at which point the material starts yielding with no further increase of the effective stress. Instead of the elastic-perfectly plastic material, any kind of other material could be used such as a strain-hardening or a strain-softening material.

In the following text, we summarize all the facts, assumptions, and solution strategy necessary to run a successful numerical simulation and obtain a reasonable air gap. 
Facts:

(1) The mold constantly expands during the entire casting process.

(2) The casting also expands but only during the early stage of casting. Later, the strength of the solidified part of the casting is sufficient to withstand centrifugal forces; therefore, the casting contracts. Consequently, the air gap forms.

Assumptions:

(1) The mold undergoes purely elastic deformations. (In reality, it may not be true, especially in the vicinity of the cast-mold interface. At the initial stage of casting, extreme stresses may occur, causing a type of damage known as "fire cracks," to the inner part of the mold.)

(2) Mechanical properties of the mold material are constant. Thermophysical properties may vary with the temperature.

(3) The casting may deform both elastically and plastically. A temperature-dependent, perfectly elastic-plastic material is assumed (Figure 2).

(4) Mechanical properties (Young's modulus $E$ and yield strength $Y$ ) of the casting material are temperature dependent. Thermophysical properties may also vary with the temperature.

(5) Only the radiative and the conductive heat transfer mechanisms are expected within the air gap. The convective mechanism is neglected due to the small size of the air gap.

In addition to Eqs. [10] and [11], we also need to solve the heat conduction for the temperature $T$. In the cylindrical coordinate system, it takes this form in the mold:

$$
\rho_{\mathrm{M}} c_{\mathrm{pM}} \frac{\partial T}{\partial t}=\frac{1}{r} \frac{\partial}{\partial r}\left(k_{\mathrm{M}} \frac{\partial T}{\partial r}\right),
$$

where $\rho_{\mathrm{M}}, c_{\mathrm{pM}}$, and $k_{\mathrm{M}}$ are the density, specific heat, and thermal conductivity of the mold material, respectively. Similarly, in the casting, it can be written as

$$
\rho_{\mathrm{S}} c_{\mathrm{pS}} \frac{\partial T}{\partial t}=\frac{1}{r} \frac{\partial}{\partial r}\left(k_{\mathrm{S}} \frac{\partial T}{\partial r}\right)+\rho_{\mathrm{S}} L_{\mathrm{f}} \frac{\partial g_{\mathrm{s}}}{\partial t},
$$

where $\rho_{S}, c_{\mathrm{pS}}$, and $k_{S}$ are density, specific heat, and thermal conductivity of the casting material, respectively. The last term is a latent heat source term due to the phase change, in which $L_{\mathrm{f}}$ and $g_{\mathrm{s}}$ represent the latent heat and the solid fraction, respectively. In the present study, a simple linear relationship is considered between the solid fraction $g_{\mathrm{s}}$ and the temperature $T$. Other relationships, however, could also be considered (e.g., the lever rule or the Gulliver-Scheil equation). The heat conduction equations, Eqs. [15] and [16], are coupled via the heat flux at the cast-mold interface. A thin-wall model, also known as a zero-capacity model, was used to numerically simplify the situation at the interface by considering only a thermal resistance, exerted by the coating and possibly the air gap. Then, the heat flux at the interface reads as

$$
\begin{gathered}
q=k_{\mathrm{ifc}} \frac{T_{\mathrm{S}}-T_{\mathrm{M}}}{d r_{\mathrm{M}}+d r_{\mathrm{S}}}, \\
k_{\mathrm{ifc}}=\frac{k_{\mathrm{S}} k_{\mathrm{M}} k_{\mathrm{C}} k_{\mathrm{a}}\left(d r_{\mathrm{M}}+d r_{\mathrm{S}}\right)}{\left(k_{\mathrm{C}} k_{\mathrm{a}}\left(d r_{\mathrm{M}} k_{\mathrm{S}}+d r_{\mathrm{S}} k_{\mathrm{M}}\right)+k_{\mathrm{S}} k_{\mathrm{M}}\left(d_{\mathrm{C}} k_{\mathrm{a}}+d_{\mathrm{a}} k_{\mathrm{C}}\right)\right)},
\end{gathered}
$$

where $k_{\text {ifc }}, k_{\mathrm{C}}$, and $k_{\mathrm{a}}$ denote the effective thermal conductivity, the thermal conductivity of the coating, and the air gap, respectively. Other quantities are explained in Figure 1. The air gap thermal conductivity $k_{\mathrm{a}}$ is, in fact, the sum of the thermal conductivity of air $k_{\mathrm{a} \text {,phys }}$ and a thermal conductivity, which is equivalent to the radiative heat transfer through the air gap, given by

$$
k_{\mathrm{a}}=k_{\mathrm{a}, \text { phys }}+\sigma d_{\mathrm{a}}\left(T_{\mathrm{S}}^{\prime}+T^{*}\right)\left(T_{\mathrm{S}}^{2}+T^{* 2}\right),
$$

where $\sigma$ is the Stefan-Boltzman constant $\left(5.67 \times 10^{-8} \mathrm{~W} \mathrm{~m}^{-2} \mathrm{~K}^{-4}\right)$. The temperatures $T^{\prime}$, and $T^{*}$ must be given in Kelvin. Note that without the air gap $\left(d_{\mathrm{a}}=0\right)$, Eq. [18] is still valid and represents the effective thermal conductivity only in the presence of the coating. The reader should be reminded that in this study, the black body radiation model has been taken for its simplicity and convenience. However, when targeting more accurate results, considering gray bodies is better justified and Eq. [19] would then become

$$
k_{\mathrm{a}}=k_{\mathrm{a}, \text { phys }}+\sigma d_{\mathrm{a}}\left(T_{\mathrm{S}}^{\prime}+T^{*}\right)\left(T_{\mathrm{S}}^{2}+T^{* 2}\right)\left(1 / \varepsilon_{\mathrm{S}}+1 / \varepsilon_{\mathrm{C}}-1\right)^{-1},
$$

where $\varepsilon_{\mathrm{S}}$ and $\varepsilon_{\mathrm{C}}$ are emissivity coefficients of both surfaces enclosing the air gap, which belong to the casting and the coating, respectively. Using Eq. [20]. instead of Eq. [19] will naturally reduce the radiative heat transfer. In reality, the air between the casting and the shell may, as a participating gas, further reduce the radiative heat transfer. A description of the corresponding mathematical model can be found, e.g., in Reference 52.

Concerning thermal boundary conditions, both the free surface of the casting and the outer surface of the mold are considered to be adiabatic:

$$
\begin{gathered}
\frac{\mathrm{d} T}{\mathrm{~d} r}\left(r_{i}\right)=0, \\
\frac{\mathrm{d} T}{\mathrm{~d} r}\left(r_{o}\right)=0 .
\end{gathered}
$$

In simulations focused on a comparison with experimental data, thermal boundary conditions, however, should be specified more precisely, e.g., with the help of existing empirical formulas for the Nusselt number ${ }^{[33,54]}$ in rotating geometries.

In addition to Eq. [21], boundary conditions have to be specified also for Eqs. [10] and [11]. Here, we have to distinguish between two cases: a perfect contact or an air 
gap. In the case of the contact, appropriate boundary conditions take the following form:

$$
\begin{aligned}
& \sigma_{\mathrm{rS}}\left(r_{\mathrm{i}}\right)=0 \\
& u_{\mathrm{S}}(R)=u_{\mathrm{M}}(R) \\
& \sigma_{\mathrm{rM}}\left(r_{\mathrm{o}}\right)=0 .
\end{aligned}
$$

Otherwise (the air gap),

$$
\begin{gathered}
\sigma_{\mathrm{rS}}\left(r_{\mathrm{i}}\right)=0 \\
\sigma_{\mathrm{rS}}(R)=0 \\
\sigma_{\mathrm{rM}}(R)=0 \\
\sigma_{\mathrm{rM}}\left(r_{\mathrm{o}}\right)=0 .
\end{gathered}
$$

Differential equations for total displacements, Eqs. [10] and [11], and temperature, Eqs. [15] and [16], were all solved using the finite difference method with second-order accurate central difference schemes for derivatives (including points at the boundaries). In the radial direction, the casting and the mold were divided into $N_{\mathrm{S}}$ and $N_{\mathrm{M}}$ uniformly spaced grid points with the dimensions $d r_{\mathrm{S}}$ and $d r_{\mathrm{M}}$ of $1 \mathrm{~mm}$ (Figure 1). The same grids with uniform spacing were used for both quantities $u$ and $T$. For Eqs. [15] and [16], the implicit backward Euler method was used for time-stepping. In addition, an iterative approach was necessary for heat conduction equations due to temperature-dependent thermophysical properties, the nonlinear heat flux $q$ at the cast-mold interface (Eq. [17]), and especially the stiff, latent heat source term. Treatment of the latent heat source term was realized by using a semi-implicit method proposed by Voller and Swaminathan. ${ }^{[55]}$ A detailed description of the discretization can be found in Reference 56. Although systems of equations are unconditionally stable, the time-step size should be small enough so that the loading rate still allows finding correct and physically meaningful increments of plastic strains. Here, the time-step of 0.1 seconds was found to be reasonable.

Solution strategy:

(1) Initialize fields of temperature, solid fraction, plastic strains, stresses, and air gap thickness.

(2) For time $t_{n+1}$, solve heat conduction equations, Eqs. [15] and [16], coupled by the heat flux at the interface (Eq. [17]), with the air gap thickness $d_{\mathrm{a}}$ from the previous time $t_{n}$ and obtain new temperature $T$ and solid fraction $g_{\mathrm{s}}$ fields. In our numerical tests, usually between two and five iterations were necessary to drop scaled residuals below $10 \times 10^{-8}$ for the latent heat source term and the nonlinear heat flux $q$, respectively. The residuals were calculated according to the following formula:

$$
\text { res }=\frac{\sqrt{N \sum_{i}\left(T_{i}-T_{i}^{\text {old }}\right)^{2}}}{\sum_{i} T_{i}^{\text {old }}}
$$

where $N$ is the total number of cells $\left(N_{\mathrm{S}}+N_{\mathrm{M}}\right) . T_{i}$ and $T_{i}^{\text {old }}$ are the current temperature and the temperature from the previous iteration both taken at the grid point with the index $i$

(3) Use Figures 3 and 4 to find new values of yield strength $Y$ and Young's modulus $E$ of the casting material.

(4) Assume an air gap. For time $t_{n+1}$, solve Eq. [11] for total displacements $u$ of mold with the boundary conditions for the radial stress $\sigma_{\mathrm{rM}}$ in Eq. [23]. Similarly, for time $t_{n+1}$, solve Eq. [10] for total displacements $u$ of the casting with the boundary conditions for the radial stress $\sigma_{\mathrm{rS}}$ in Eq. [23] and the plastic strains $\varepsilon_{\mathrm{r}}^{\mathrm{p}}$ and $\varepsilon_{\mathrm{t}}^{\mathrm{p}}$ from the previous time $t_{n}$.

(5) If the total displacement of the casting at the interface is greater than that of the mold, the casting and the mold are in perfect contact, i.e., no air gap is formed. Otherwise, the cast-mold contact is lost and the air gap is formed. If the earlier is true (perfect contact), recalculate Eqs. [10] and [11] with the boundary conditions given in Eq. [22]. Otherwise, evaluate the air gap thickness as

$$
d_{\mathrm{a}}=u_{\mathrm{M}}(R)-u_{\mathrm{S}}(R)
$$

(6) In the casting, evaluate radial and tangential stresses by using the following explicit formulas, which can be obtained by straightforward manipulations of Eqs. [8] and [9]:

$$
\begin{aligned}
& \sigma_{\mathrm{rS}}=\frac{E}{1-v^{2}}\left[\left(\frac{\mathrm{d} u}{\mathrm{~d} r}-\varepsilon_{r}^{p}\right)+v\left(\frac{u}{r}-\varepsilon_{t}^{p}\right)\right]-\alpha E \frac{1}{1-v} T, \\
& \sigma_{\mathrm{tS}}=\frac{E}{1-v^{2}}\left[\left(\frac{u}{r}-\varepsilon_{t}^{p}\right)+v\left(\frac{\mathrm{d} u}{\mathrm{~d} r}-\varepsilon_{r}^{p}\right)\right]-\alpha E \frac{1}{1-v} T .
\end{aligned}
$$

(7) Using Eq. [26] in Eq. [12], calculate von Mises stresses $\bar{\sigma}$ in the casting and compare them with yield stresses $Y$ obtained from the stress-strain curve (Figure 3 ). Then, identify only the points $P$ that yield $(P=\bar{\sigma} \geq Y)$.

(8) For the points $P$, new increments $\overline{d \varepsilon^{p}}$ of the effective plastic strain $\overline{\varepsilon^{p}}$ must be calculated so that

$$
\bar{\sigma}-Y=0
$$

This is realized through an optimization loop, in which Eq. [27] is the objective function and $\overline{d \varepsilon^{\mathrm{p}}}$ is constrained to values greater than zero. Then, one iteration sequence could have the following form: Estimate increments $\overline{d \varepsilon^{p}}$; using Eqs. [13] and [14], calculate increments $d \varepsilon_{r}^{p}$ and $d \varepsilon_{t}^{p}$ and update the plastic strains $\varepsilon_{r}^{p}$ and $\varepsilon_{t}^{p}\left(\varepsilon_{r}^{p}=\varepsilon_{r}^{p}+d \varepsilon_{r}^{p}, \varepsilon_{t}^{p}=\varepsilon_{t}^{p}+d \varepsilon_{t}^{p}\right)$; solve Eq. [10] for new displacements $u$ in the casting and get new stresses (Eq. [26]); and recalculate von Mises stresses $\bar{\sigma}$ and repeat until the convergence of Eq. [27] is attained. In the present article, a nonlinear least-squares optimization algorithm, known as the trust-region-reflective algorithm, ${ }^{[57,58]}$ was applied. In order to reduce dispersive errors of $\overline{d \varepsilon^{p}}$ appearing due to complex loading and, consequently, yielding on a discrete grid, the Savitzky-Golay filter ${ }^{[59]}$ was applied on a temporally overlaid $\overline{d \varepsilon^{p}}$ signal.

(9) Proceed to the next time-step. 


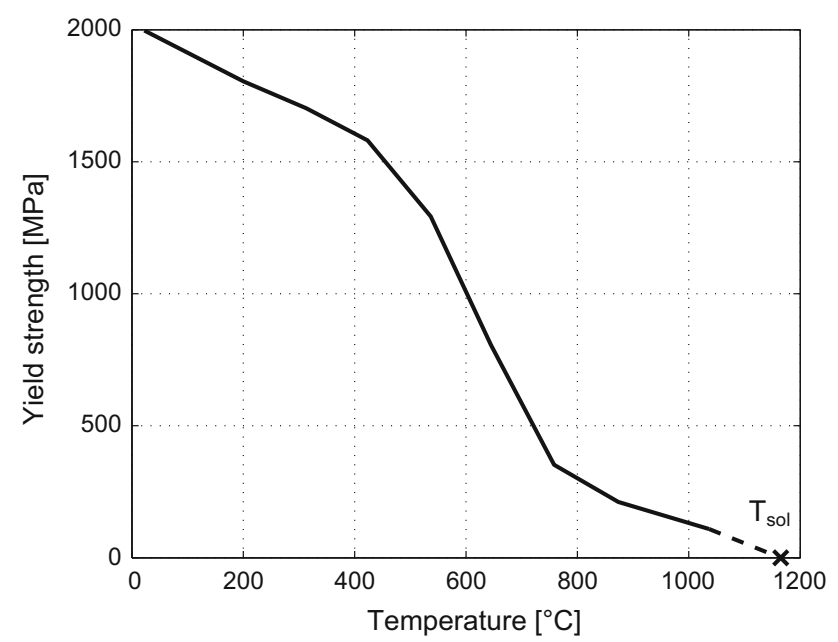

Fig. 3-Temperature-dependent yield strength of the casting material. ${ }^{[60]}$ The dashed line represents data reconstructed using the extrapolation until the temperature of solidus $T_{\text {sol }}$.

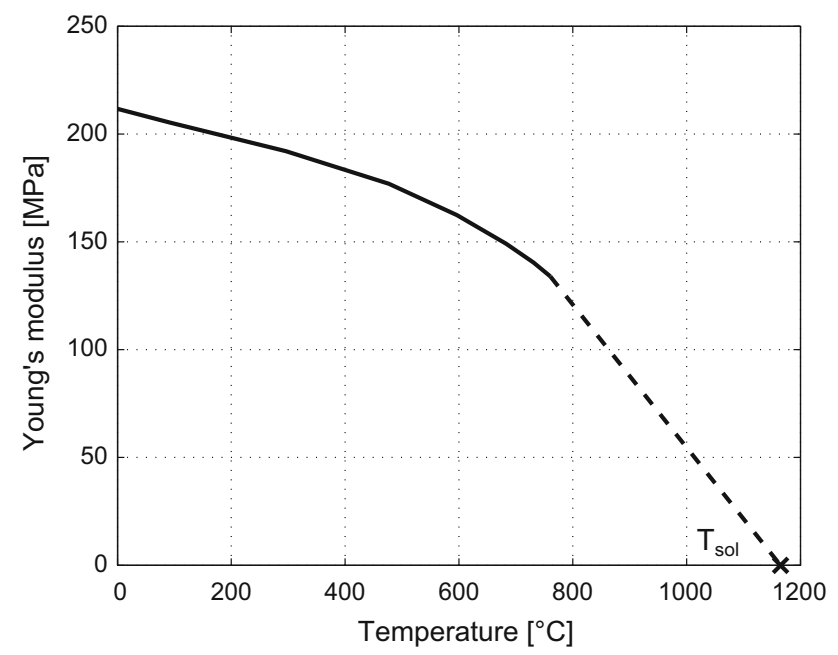

Fig. 4-Temperature-dependent Young's modulus of the casting material. ${ }^{[60]}$ The dashed line represents data reconstructed by means of extrapolation until the solidus temperature $T_{\mathrm{sol}}$.

\section{RESULTS AND DISCUSSION}

As the main objective of the present study is to propose and test a novel approach of calculating an air gap rather than numerically analyzing a particular process, material and mechanical properties used in the simulations only roughly correspond to those of real materials (Table II). The geometry, initial conditions, and other casting parameters are given in Table III.

First, numerical tests were performed for different coating thicknesses $(0.5,1,2$, and $4 \mathrm{~mm})$ and all other parameters were fixed. Total displacements $u$ of the mold and the casting at the interface are shown as a function of time $t$ in Figure 5. As one would expect, initially, the mold and the casting are in contact. At a certain moment, they detach and their displacements follow different paths. This is clearly shown by bifurcating curves in Figure 5. A difference between the casting and the mold displacement corresponds to the
Table II. Properties of Materials Used in the Simulations

\begin{tabular}{|c|c|c|c|}
\hline Material & Property & Value & Unit \\
\hline \multirow[t]{11}{*}{ Casting $[\ldots]_{S}$} & $\alpha$ & $5 \times 10^{-6}$ & $\mathrm{~K}^{-1}$ \\
\hline & $\rho$ & 7860 & $\mathrm{~kg} \mathrm{~m}^{-3}$ \\
\hline & $c_{\mathrm{p}}$ & 500 & $\mathrm{~J} \mathrm{~kg}^{-1} \mathrm{~K}^{-1}$ \\
\hline & $v$ & 0.5 & - \\
\hline & $E$ & Fig. 4 & $\mathrm{~Pa}$ \\
\hline & $g_{\mathrm{s}}$ & linear between $T_{\text {liq }}$ and $T_{\text {sol }}$ & - \\
\hline & $k$ & 22 & $\mathrm{~W} \mathrm{~m}^{-1} \mathrm{~K}^{-1}$ \\
\hline & $L_{\mathrm{f}}$ & 280 & $\mathrm{~kJ} \mathrm{~kg}^{-1}$ \\
\hline & $T_{\text {liq }}$ & $1593(1320)$ & $\mathrm{K}\left({ }^{\circ} \mathrm{C}\right)$ \\
\hline & $T_{\text {sol }}$ & $1438(1165)$ & $\mathrm{K}\left({ }^{\circ} \mathrm{C}\right)$ \\
\hline & $Y$ & Fig. 3 & $\mathrm{~Pa}$ \\
\hline \multirow[t]{6}{*}{ Mold $[\ldots]_{M}$} & $\alpha$ & $5 \times 10^{-6}$ & $\mathrm{~K}^{-1}$ \\
\hline & $v$ & 0.5 & - \\
\hline & $\rho$ & 7850 & $\mathrm{~kg} \mathrm{~m}^{-3}$ \\
\hline & $c_{\mathrm{p}}$ & 490 & $\mathrm{~J} \mathrm{~kg}^{-1} \mathrm{~K}^{-1}$ \\
\hline & E & $200 \times 10^{9}$ & $\mathrm{~Pa}$ \\
\hline & $k$ & 58.6 & $\mathrm{~W} \mathrm{~m} \mathrm{~m}^{-1} \mathrm{~K}^{-1}$ \\
\hline Coating & $k_{\mathrm{C}}$ & 2 & $\mathrm{~W} \mathrm{~m} \mathrm{~m}^{-1} \mathrm{~K}^{-1}$ \\
\hline Air & $k_{\mathrm{a}, \mathrm{phys}}$ & 0.02 & $\mathrm{~W} \mathrm{~m}^{-1} \mathrm{~K}^{-1}$ \\
\hline
\end{tabular}

Table III. Geometry, Initial Conditions, and Other Casting Parameters

\begin{tabular}{lll}
\hline Property & \multicolumn{1}{c}{ Value } & Unit \\
\hline$\Omega$ & 71 & $\mathrm{rad} \mathrm{s}^{-1}$ \\
$d$ & 65 & $\mathrm{~mm}$ \\
$d_{\mathrm{C}}$ & 2 & $\mathrm{~mm}$ \\
$L$ & 165 & $\mathrm{~mm}$ \\
$R$ & 400 & $\mathrm{~mm}$ \\
$T_{\text {fill }}$ & $1623(1350)$ & $\mathrm{K}\left({ }^{\circ} \mathrm{C}\right)$ \\
$T_{\text {mold }}$ & $433(160)$ & $\mathrm{K}\left({ }^{\circ} \mathrm{C}\right)$ \\
\hline
\end{tabular}

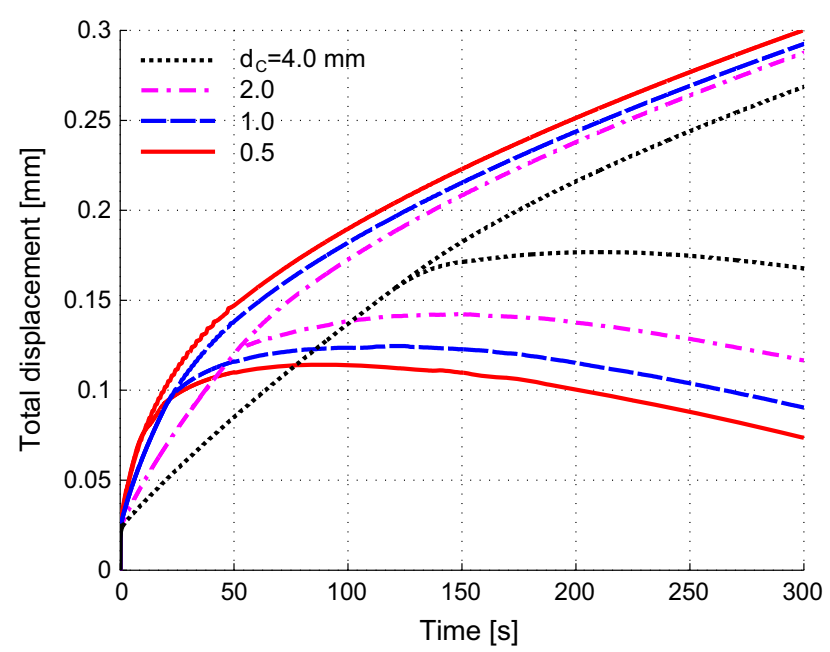

Fig. 5-Total displacements $u$ at the cast-mold interface for different coating thicknesses of $0.5,1,2$, and $4 \mathrm{~mm}$. Bifurcation corresponds to the first appearance of the air gap.

air gap thickness $d_{\mathrm{a}}$. Obviously, the air gap appears earlier in the case of a thin coating than that of a thick one. Consequently, the heat transfer coefficient $h$ at the 
interface will drop faster in the case of a thin coating than that of a thick one, which can be seen in Figure 6 . The heat transfer coefficient $h$ was simply calculated as

$$
h=\frac{q}{T_{\mathrm{S}}-T_{\mathrm{M}}}=\frac{k_{\mathrm{ifc}}}{d r_{\mathrm{M}}+d r_{\mathrm{S}}} .
$$

For each configuration $\left(d_{\mathrm{C}}=0.5,1,2\right.$, and $\left.4 \mathrm{~mm}\right)$, the time evolution of the heat transfer coefficient $h$ is, therefore, unique. Ultimately, it seems that after 300 seconds, the heat transfer coefficients are almost identical, close to $200 \mathrm{~W} \mathrm{~m}^{-2} \mathrm{~K}^{-1}$.

Similar tests with similar outputs were performed for different values of the rotation rate $\Omega(50,71$, and $90 \mathrm{rad}$ $\mathrm{s}^{-1}$ ). Naturally, the higher the centrifugal force, the better the contact between the casting and the mold. Concerning the elastic deformations of the mold, displacements $u$ are larger at higher rotation rates $\Omega$ (Figure 7). Higher centrifugal forces are also responsible for stronger and longer yielding of the partly solidified casting, which delays a formation of the air gap. Consequently, at a given instance, the heat transfer coefficient $h$ is higher in the case of a higher rotation rate $\Omega$ (Figure 8).

A similar study was carried out for different values of solidus temperature $T_{\text {sol }}$ such that $T_{\text {liq }}-T_{\text {sol }}=10 \mathrm{~K}$, $50 \mathrm{~K}, 100 \mathrm{~K}$, and $200 \mathrm{~K}\left(10{ }^{\circ} \mathrm{C}, 50{ }^{\circ} \mathrm{C}, 100{ }^{\circ} \mathrm{C}\right.$, and 200 ${ }^{\circ} \mathrm{C}$ ). Although it is a somewhat intuitive, one could confidently state that the smaller the difference is, the earlier the air gap occurs. Again, we provide total displacements and heat transfer coefficients at the interface in Figures 9 and 10, respectively. Since coating parameters are fixed this time, initial heat transfer coefficients are all identical, equal to $1000 \mathrm{~W} \mathrm{~m}^{-2} \mathrm{~K}^{-1}$. Later, they significantly deviate. While the curves referring to mold displacements at the interface have a similar trend, indicating a continuous thermoelastic expansion of the mold, those corresponding to casting displacements exhibit more complex scenarios due to the combination of plastic and elastic deformations. In

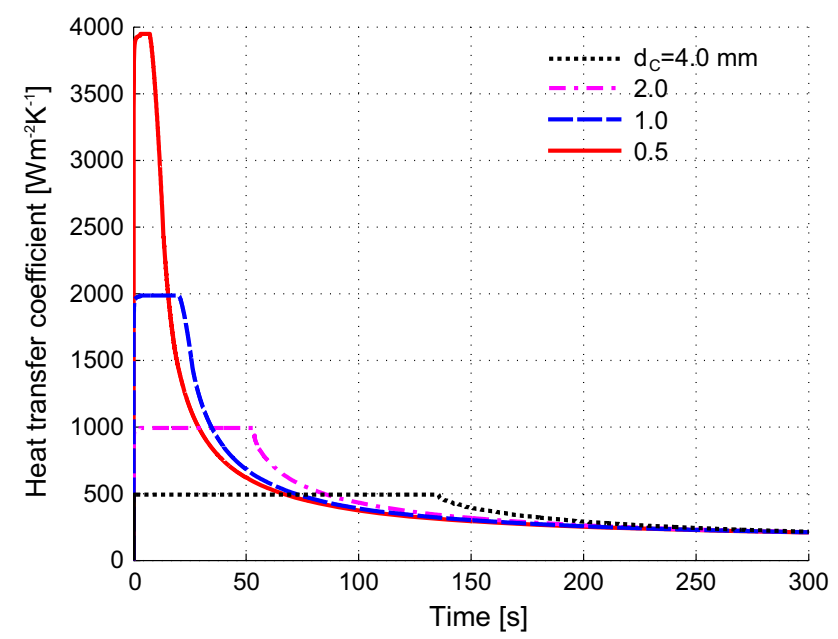

Fig. 6-Heat transfer coefficients $h$ at the cast-mold interface for different coating thicknesses of $0.5,1,2$, and $4 \mathrm{~mm}$. Before the air gap is formed, $h$ is constant defined as $k_{\mathrm{C}} d_{\mathrm{C}}$.
Figure 9, e.g., the dash-dot line representing $T_{\text {liq }}-T_{\text {sol }}$ $=200 \mathrm{~K}\left(200{ }^{\circ} \mathrm{C}\right)$ indicates the yielding of the casting material within the entire time span simulated. On the contrary, the dash line $T_{\text {liq }}-T_{\text {sol }}=50 \mathrm{~K}\left(50{ }^{\circ} \mathrm{C}\right)$ displays only slight yielding in the beginning, immediately followed by thermoelastic contraction.

Although the quantities calculated at the interface, such as the air gap thickness $d_{\mathrm{a}}$ and the heat transfer coefficient $h$, are of primary interest here, the numerical model also provides other quantities such as stresses and elastic/plastic strains. In Figure 11, a typical example of temperature and strains appears at 50 seconds. The gray zone on the right represents the mold. The rest on the left belongs to the casting. A temperature drop can be seen at the interface due to a large thermal resistance. As the time proceeds, total strains grow quite uniformly throughout the entire thickness of the mold. The same applies also to the casting but only at the early stage. Later, when the casting is partly solidified and the yield

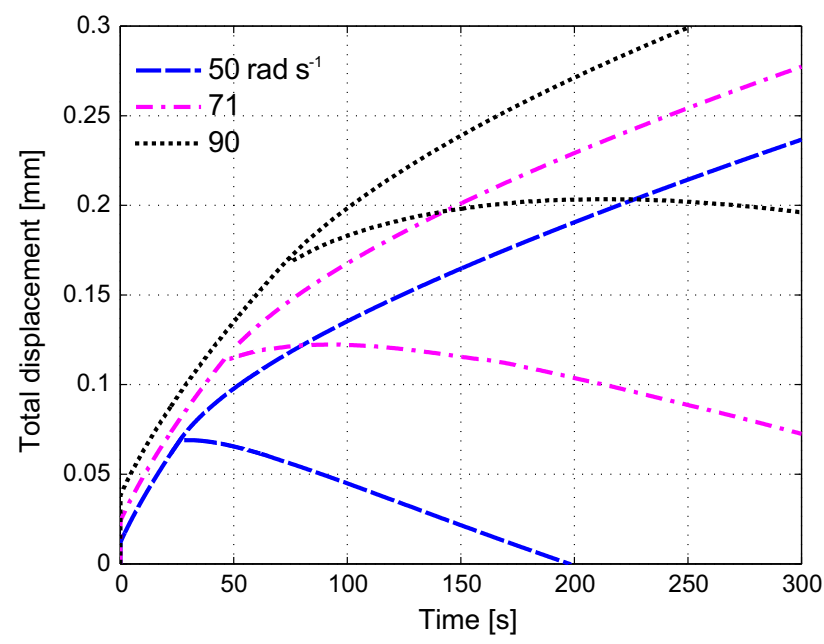

Fig. 7-Total displacements $u$ at the cast-mold interface for different values of rotation rate $\Omega$ such that $\Omega=50,71,90 \mathrm{rad} \mathrm{s}^{-1}$.

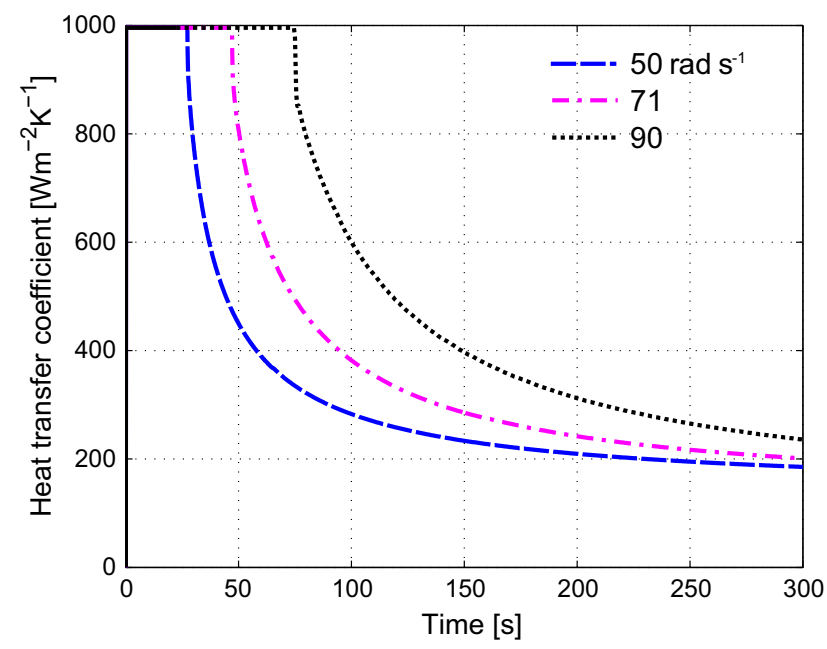

Fig. 8-Heat transfer coefficients $h$ at the cast-mold interface for different values of rotation rate $\Omega$ such that $\Omega=50,71$, and $90 \mathrm{rad}$ $\mathrm{s}^{-1}$. 


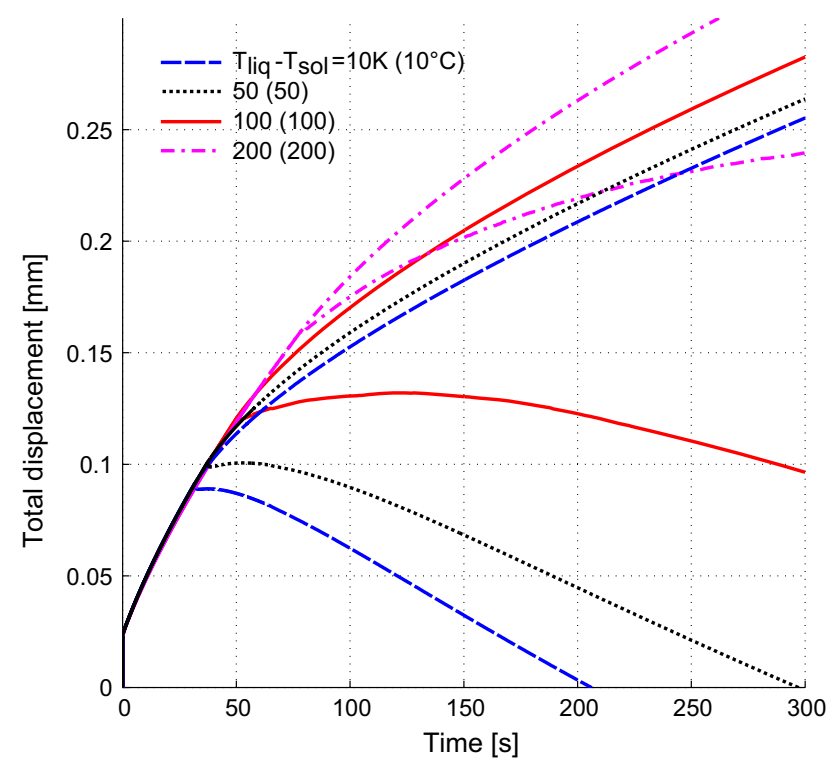

Fig. 9-Total displacements $u$ at the cast-mold interface for different values of solidus temperature $T_{\text {sol }}$ such that $T_{\text {liq }}-T_{\text {sol }}=10 \mathrm{~K}, 50$ $\mathrm{K}, 100 \mathrm{~K}$, and $200 \mathrm{~K}\left(10^{\circ} \mathrm{C}, 50^{\circ} \mathrm{C}, 100^{\circ} \mathrm{C}\right.$, and $\left.200^{\circ} \mathrm{C}\right)$.

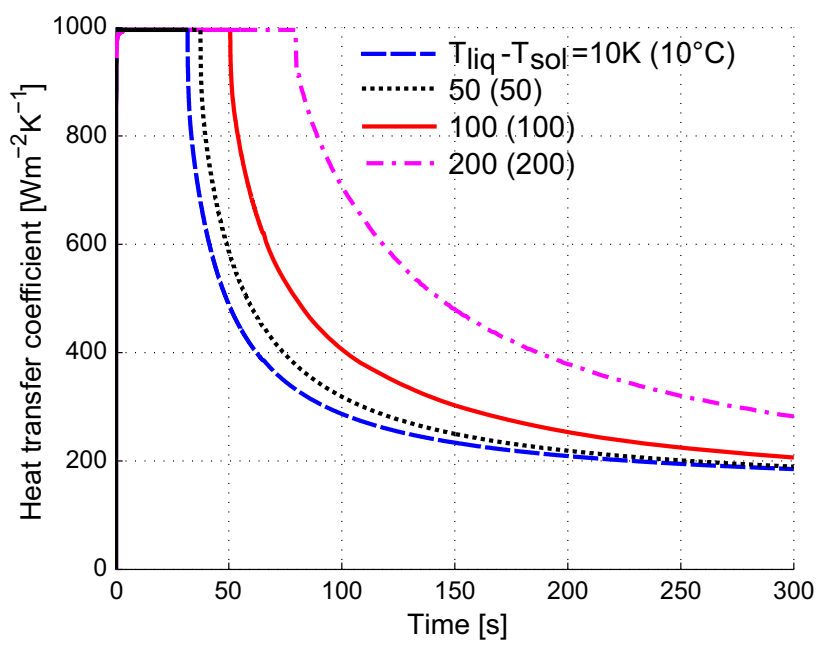

Fig. 10-Heat transfer coefficients $h$ at the cast-mold interface for different values of solidus temperature $T_{\text {sol }}$ such that $T_{\text {liq }}-T_{\text {sol }}=$ $10 \mathrm{~K}, 50 \mathrm{~K}, 100 \mathrm{~K}$, and $200 \mathrm{~K}\left(10^{\circ} \mathrm{C}, 50^{\circ} \mathrm{C}, 100^{\circ} \mathrm{C}\right.$, and $\left.200^{\circ} \mathrm{C}\right)$.

strength $Y$ increased, total strains start dropping and the casting contracts consequently.

In addition to strains (Figure 11), stresses are shown in Figure 12. A typical distribution of stresses can be seen in the mold. While the radial stresses are exclusively compressive, the tangential stresses are compressive close to the inner surface of the mold and become tensile as they approach the outer surface of the mold. The greatest stresses the mold must withstand are naturally located at the inner surface due to a sudden temperature loading. In this particular case (Figure 12), they do, in

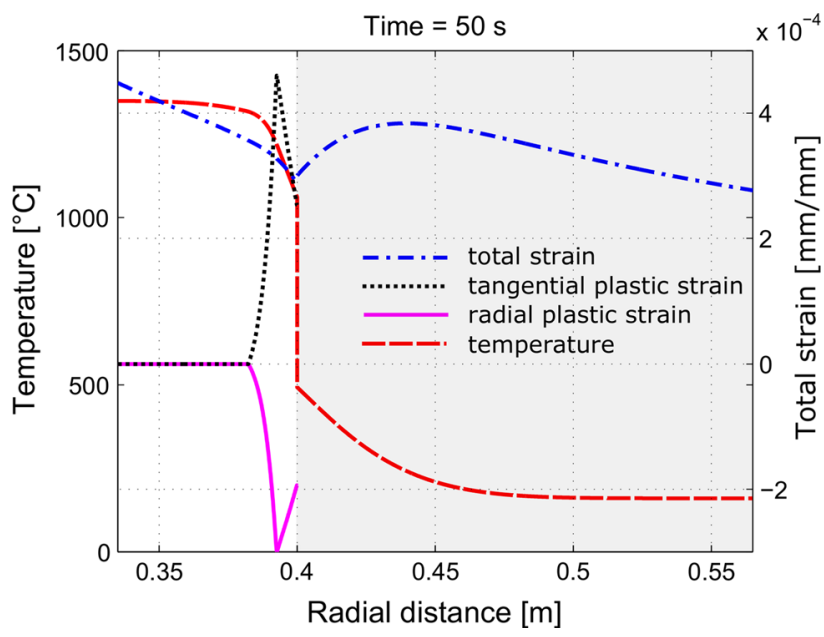

Fig. 11-Distribution of strains and temperature in the radial direction for the case with the coating thickness $d_{\mathrm{C}}$ of $2.0 \mathrm{~mm}$ at 50 $\mathrm{s}$. The zones in white and gray stand for the casting and the mold, respectively.

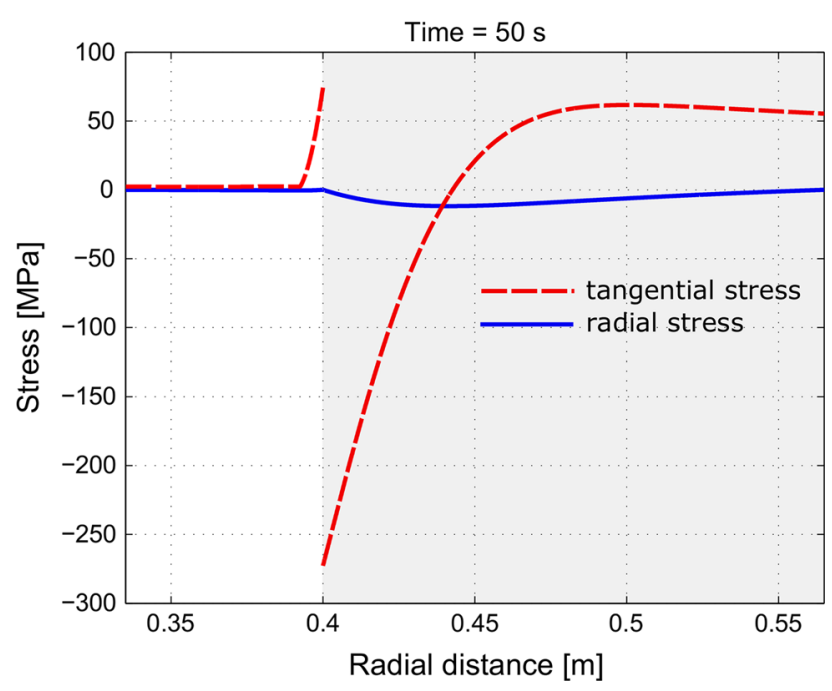

Fig. 12-Distribution of radial and tangential stresses for the case with the coating thickness $d_{\mathrm{C}}$ of $2.0 \mathrm{~mm}$ at $50 \mathrm{~s}$.

fact, reach the yield strength of the material. After several casting cycles, a thermal loading would most likely lead to the formation of fire cracks. ${ }^{[61]}$ Concerning stresses in the casting, at the early stage of solidification, they are within the temperature-dependent envelope of the yield strength. The (semi-)solid part of the casting close to the mold can already hold some stresses, whereas the liquid part remains stress free. At later stages of the casting, the stresses in the casting are well below the yield strength and only the elastic loading is present. When the casting procedure is finished and the temperature field becomes uniform, residual stresses remain in the casting. An analysis of stresses and strains is, however, beyond the scope of the present article. 


\section{CONCLUSIONS}

In centrifugal casting simulations, exponential functions are generally used to describe the heat transfer coefficient at the cast-mold interface, varying due to the air gap formation. Such functions contain empirical constants, which must be carefully specified. Unfortunately, this is not an easy task. An experiment alone is not sufficient to determine such constants, and computationally expensive inverse methods should be employed, which is, however, rarely the case. A literature survey performed here reveals an expansive scatter of data used in current and previous research. In the present study, we offer an alternative of calculating an air gap thickness and the corresponding heat transfer coefficient at the interface. The heat transfer model is coupled with a plane stress model, taking into account thermoelastic stresses, centrifugal forces, plastic deformations, and a temperature-dependent Young's modulus. Several numerical tests were performed for different coating thicknesses $d_{\mathrm{C}}$, rotation rates $\Omega$, and solidus temperatures $T_{\text {sol }}$. Results were analyzed in the sense of comparing heat transfer coefficients at the interface and air gap thicknesses as a function of time. The numerical model developed here helps demonstrate that the scenario at the interface is unique for each set of parameters. Therefore, deploying any of the exponential functions that explicitly describe the thermal resistance at the cast-mold interface will always give rise to the question about the actual value of empirical constants used in that particular function. Although the material properties taken for this study do not strictly correspond to any particular material, they are obviously not far from material properties of common steels and coatings, and the results obtained here appear to be entirely reasonable and meaningful. In the near future, we plan to verify the current numerical approach against the results obtained from the inverse task run with the experimental data. Finally, possible room for improvement of the presented model remains. For example, some kind of implicit coupling between the heat transfer model and the plane stress model would be beneficial and might even be necessary in order to maintain numerical stability (or suppress unphysical oscillations of calculated displacements), especially at higher cooling rates, e.g., when an air gap is being just formed. In addition, the optimization loop involved in the loading step, i.e., the process of calculating the plastic strains could also be further improved.

\section{ACKNOWLEDGMENTS}

Open access funding provided by Montanuniversity Leoben. Financial support from the Austrian Federal Government (in particular, from the Bundesministerium fuer Verkehr, Innovation und Technologie and the Bundesministerium fuer Wirtschaft, Familie und Jugend) and the Styrian Provincial Government, represented by Oesterreichische Forschungsfoerderungsgesellschaft
$\mathrm{mbH}$ and by Steirische Wirtschaftsfoerderungsgesellschaft $\mathrm{mbH}$, within the research activities of the K2 Competence Centre on "Integrated Research in Materials, Processing and Product Engineering," operated by the Materials Center Leoben Forschung GmbH in the framework of the Austrian COMET Competence Centre Programme, is gratefully acknowledged. This work is also financially supported by the Eisenwerk Sulzau-Werfen R. \& E. Weinberger AG.

\section{OPEN ACCESS}

This article is distributed under the terms of the Creative Commons Attribution 4.0 International License (http://creativecommons.org/licenses/by/4.0/), which permits unrestricted use, distribution, and reproduction in any medium, provided you give appropriate credit to the original author(s) and the source, provide a link to the Creative Commons license, and indicate if changes were made.

\section{NOMENCLATURE}

$[\ldots] C \quad$ Subscript referring to the coating

$[\ldots] M \quad$ Subscript referring to the mold

$[\ldots]_{S} \quad$ Subscript referring to the casting

$c_{\mathrm{p}} \quad$ Specific heat $\left(\mathrm{J} \mathrm{kg}^{-1} \mathrm{~K}^{-1}\right)$

$d \quad$ Casting thickness $(\mathrm{mm})$

$d_{\mathrm{a}} \quad$ Air gap thickness (mm)

$d_{\mathrm{C}} \quad$ Coating thickness $(\mathrm{mm})$

E Young's modulus $(\mathrm{Pa})$

$g_{\mathrm{s}} \quad$ Solid fraction

$h \quad$ Heat transfer coefficient at the interface $\left(\mathrm{W} \mathrm{m}^{-2} \mathrm{~K}^{-1}\right.$ )

$h_{0} \quad$ Initial heat transfer coefficient at the interface $\left(\mathrm{W} \mathrm{m} \mathrm{m}^{-2} \mathrm{~K}^{-1}\right.$ )

$h_{\mathrm{f}} \quad$ Final heat transfer coefficient at the interface $\left(\mathrm{W} \mathrm{m}{ }^{-2} \mathrm{~K}^{-1}\right.$ )

$h_{\mathrm{a}} \quad$ Heat transfer coefficient between the casting and the coating $\left(\mathrm{W} \mathrm{m}^{-2} \mathrm{~K}^{-1}\right)$

$k \quad$ Thermal conductivity $\left(\mathrm{W} \mathrm{m}^{-1} \mathrm{~K}^{-1}\right.$ )

$k_{\mathrm{a}} \quad$ Effective thermal conductivity of the air gap $\left(\mathrm{W} \mathrm{m}^{-1} \mathrm{~K}^{-1}\right.$ )

$k_{\text {a,phys }} \quad$ Thermal conductivity of air $\left(\mathrm{W} \mathrm{m}^{-1} \mathrm{~K}^{-1}\right)$

$k_{\mathrm{C}} \quad$ Thermal conductivity of the coating (W m ${ }^{-1} \mathrm{~K}^{-1}$ )

$k_{\text {ifc }} \quad$ Effective thermal conductivity of the control volume built of the mold, coating, air gap, and casting $\left(\mathrm{W} \mathrm{m}^{-1} \mathrm{~K}^{-1}\right)$

$L \quad$ Thickness of the mold (m)

$L_{\mathrm{f}} \quad$ Latent heat of solidification $\left(\mathrm{J} \mathrm{kg}^{-1}\right)$

$N \quad$ Number of grid points

$P \quad$ Set of yielding points

$q \quad$ Heat flux through the interface $\left(\mathrm{W} \mathrm{m}^{-2}\right)$

$q_{0} \quad$ Initial heat flux through the interface (only coating present $)\left(\mathrm{W} \mathrm{m}^{-2}\right)$

$r \quad$ Radial distance $(\mathrm{mm})$

$r_{\mathrm{i}} \quad$ Inner radius of the casting $(\mathrm{mm})$

$r_{\mathrm{o}} \quad$ Outer radius of the mold $(\mathrm{mm})$ 
Inner radius of the mold-radius of the interface $(\mathrm{mm})$

Solidified thickness of the casting $(\mathrm{mm})$

Time (s)

Time of solidification initiation (s)

Temperature $\left(\mathrm{K}\left({ }^{\circ} \mathrm{C}\right)\right)$

$T$

$T_{\text {fill }}$

Initial temperature of the liquid metal-filling temperature $\left(\mathrm{K}\left({ }^{\circ} \mathrm{C}\right)\right)$

$T_{\text {liq }} \quad$ Liquidus temperature of the casting material $\left(\mathrm{K}\left({ }^{\circ} \mathrm{C}\right)\right)$

$T_{\text {mold }} \quad$ Initial temperature of the mold $\left(\mathrm{K}\left({ }^{\circ} \mathrm{C}\right)\right)$

$T_{\text {sol }} \quad$ Solidus temperature of the casting material (K $\left.\left({ }^{\circ} \mathrm{C}\right)\right)$

$T^{*} \quad$ Temperature between the coating and the air gap (Fig. 1) $\left(\mathrm{K}\left({ }^{\circ} \mathrm{C}\right)\right)$

$T^{\prime}{ }_{\mathrm{M}} \quad$ Temperature between the mold and the coating $\left(\mathrm{K}\left({ }^{\circ} \mathrm{C}\right)\right)$

$T^{\prime}$ S Temperature between the casting and the air gap $\left(\mathrm{K}\left({ }^{\circ} \mathrm{C}\right)\right)$

$u \quad$ Radial displacement $(\mathrm{mm})$

$Y \quad$ Yield strength $(\mathrm{Pa})$

\section{GREEK SYMBOLS}

$\alpha \quad$ Thermal expansion coefficient $\left(\mathrm{K}^{-1}\right)$

$\beta \quad$ Damping coefficient

$\gamma \quad$ Constant exponent

$\varepsilon_{\mathrm{r}} \quad$ Total strains in radial direction $\left(\mathrm{mm} \mathrm{mm}^{-1}\right)$

$\varepsilon_{\mathrm{t}} \quad$ Total strains in tangential direction $\left(\mathrm{mm} \mathrm{mm}^{-1}\right)$

$\varepsilon_{\mathrm{r}}^{\mathrm{p}} \quad$ Plastic strains in radial direction $\left(\mathrm{mm} \mathrm{mm}^{-1}\right)$

Plastic strains in tangential direction $\left(\mathrm{mm} \mathrm{mm}^{-1}\right)$

Effective plastic strain $\left(\mathrm{mm} \mathrm{mm}^{-1}\right)$

Radiative emissivity

Poisson's ratio

Density $\left(\mathrm{kg} \mathrm{m}^{-3}\right)$

$\sigma_{\mathrm{r}} \quad$ Radial stress $(\mathrm{Pa})$

$\sigma_{\mathrm{t}} \quad$ Tangential stress $(\mathrm{Pa})$

$\bar{\sigma} \quad$ von Mises (effective stress) $(\mathrm{Pa})$

$\sigma \quad$ Stefan-Boltzmann constant $\left(\mathrm{W} \mathrm{m} \mathrm{m}^{-2} \mathrm{~K}^{-4}\right)$

$\Omega \quad$ Rate of rotation $\left(\operatorname{rad~s}^{-1}\right)$

\section{REFERENCES}

1. H. Esaka, K. Kawai, H. Kaneko, and K. Shinozuka: IOP Conference Series: Materials Science and Engineering, 2012, vol. 33, p. 012041

2. S. Wei: ASM Int., 2008, vol. 15, pp. 667-73.

3. P. Kapranos, C. Carney, A. Pola, and M. Jolly: Compr. Mater. Process., 2014, vol. 5, pp. 39-67.

4. M. Yuan, L. Cao, Y. Xu, and X. Song: Model. Num. Simul. Mater. Sci., 2014, vol. 4, pp. 20-24.

5. E. Kaschnitz: IOP Conference Series: Materials Science and Engineering, 2012, vol. 33, p. 012031.

6. T.C. Lebeau: Master's Thesis, University of Alabama, Birmingham, AL, 2008, p. 35.

7. K.S. Keerthi Prasad, M.S. Murali, and P.G. Mukunda: Front. Mater. Sci. China, 2010, vol. 4, pp. 103-11.

8. S.-L. Lu, F.-R. Xiao, S.-J. Zhang, Y.-W. Mao, and B. Liao: Appl. Therm. Eng., 2014, vol. 73, pp. 512-21.

9. D. McBride, N.J. Humphreys, T.N. Croft, N.R. Green, M. Cross, and P. Withey: Comput. Fluids, 2013, vol. 82, pp. 63-72.
10. J. Bohacek, A. Kharicha, A. Ludwig, and M. Wu: ISIJ Int., 2014, vol. 54, pp. 266-74.

11. J. Bohacek, A. Kharicha, A. Ludwig, and M. Wu: Appl. Math. Comput., 2015, vol. 267, pp. 179-94.

12. N. Song, Y. Luan, Y. Bai, Z.A. Xu, X. Kang, and D. Li: J. Mater. Sci. Technol., 2012, vol. 28, pp. 147-54.

13. H. Fu, Q. Xiao, and J. Xing: Mater. Sci. Eng. A, 2008, vol. 479, pp. $253-60$.

14. L. Drenchev, J. Sobczak, S. Malinov, and W. Sha: Model. Simul. Mater. Sci. Eng., 2003, vol. 11, pp. 651-74.

15. J.A. Hines: Metall. Mater. Trans. B, 2004, vol. 35B, pp. 299-311.

16. W.D. Griffiths and R. Kayikci: J. Mater. Sci., 2007, vol. 42, pp. $4036-43$.

17. C.P. Hallam and W.D. Griffiths: Metall. Mater. Trans. B, 2004, vol. 35B, pp. 721-33.

18. Z. Xu, N. Song, R.V. Tol, Y. Luan, and D. Li: IOP Conference Series: Materials Science and Engineering, 2012, vol. 33, p. 012030.

19. J.W. Gao and C.Y. Wang: Mater. Sci. Eng. A, 2000, vol. 292, pp. 207-15.

20. K. Cook, B. Wu, and R.G. Reddy: Int. J. Manuf. Sci. Prod., 2006, vol. 17 , pp. $48-59$.

21. J. Bohacek, A. Kharicha, A. Ludwig, and Wu M: IOP Conference Series: Materials Science and Engineering, 2012, vol. 33, p. 012032.

22. N.J. Humphreys, D. McBride, D.M. Shevchenko, T.N. Croft, P. Withey, N.R. Green, and M. Cross: Appl. Math. Model., 2013, vol. 37 , pp. $7633-43$.

23. S.R. Chang, J.M. Kim, and C.P. Hong: ISIJ Int., 2001, vol. 41, pp. 738-47.

24. CG. Kang, P.K. Rohatgi, C.S. Narendranath, and G.S. Cole: ISIJ Int., 1994, vol. 34, pp. 247-54.

25. C.G. Kang and P.K. Rohatgi: Metall. Mater. Trans. B, 1996, vol. 27B, pp. 277-85.

26. Y. Ebisu: AFS Trans., 1977, pp. 643-54.

27. Kamlesh: Ph.D. Thesis, BVMEngineering College, Gujarat, India, 2001, p. 35.

28. L. Lajoye, and M. Suery: Proc. Int. Symp. on Advances in Cast Reinforced Metal Composites, ASM International, Chicago, IL, 1988, pp. 15-20.

29. P.S.S. Raju and S.P. Mehrotra: Mater. Trans. JIM, 2000, vol. 41, pp. 1626-35.

30. L. Drenchev, J. Sobczak, S. Malinov, and W. Sha: Model. Simul. Mater. Sci. Eng., 2003, vol. 11, pp. 635-49.

31. E. Panda, D. Mazumdar, and S.P. Mehrotra: Metall. Mater. Trans. A, 2006, vol. 37A, pp. 1675-87.

32. L. Nastac: ISIJ Int., 2014, vol. 54 (6), pp. 1294-1303.

33. S. Vacca, M.A. Martorano, R. Heringer, and M. Boccalini, Jr: Metall. Mater. Trans. A, 2015, vol. 46A, pp. 2238-48.

34. F. Susac, K. Ohura, M. Banu, and A. Makinouchi: VCAD System Res., 2009, pp. 69-70.

35. H.M. Sahin, K. Kocatepe, R. Kayikci, and N. Akar: Energy Convers. Manag., 2006, vol. 47, pp. 19-34.

36. A.F. Ilkhchy, N. Varahraam, and P. Davami: Iran. J. Mater. Sci. Eng., 2012, vol. 9, pp. 11-20.

37. J.V. Beck, B. Blackwell, and C. Clair: Inverse Heat Conduction: Ill-Posed Problems, Wiley, New York, NY, 1985.

38. J. Mahmoudi: Int. J. Cast. Met. Res., 2006, vol. 19, pp. 223-36.

39. B. Coates and S.A. Argyropoulos: Metall. Mater. Trans. B, 2007, vol. 38 B, pp. $243-55$.

40. MA. Taha, N.A. El-Mahallawy, M.T. El-Mestekawi, and A.A. Hassan: Mater. Sci. Technol., 2001, vol. 17, pp. 1093-1101.

41. J. Kron: Ph.D. Thesis, Royal Institute of Technology, Stockholm, 2004, p. 15.

42. J. Kron, A. Lagerstedt, and H. Frederiksson: Int. J. Cast. Met. Res., 2005, vol. 18, pp. 29-40.

43. A. Lagerstedt: Ph.D. Thesis, Royal Institute of Technology, Stockholm, 2004, p. 41.

44. K. Schwerdtfeger, M. Sato, and K.-H. Tacke: Metall. Mater. Trans. B, 1998, vol. 29B, pp. 1057-68.

45. J. Kron, M. Bellet, A. Ludwig, B. Pustal, J. Wendt, and H. Fredriksson: Int. J. Cast. Met. Res., 2004, vol. 17, pp. 295-310.

46. M. Trovant and S.A. Argyropoulos: Can. Metall. Q., 1998, vol. 37, pp. $185-96$.

47. R.K. Nayak and S. Sundarraj: Metall. Mater. Trans. B, 2010, vol. 41B, pp. 151-60. 
48. A. Kandil, A.A. El-Kady, and A. El-Kafrawy: Int. J. Mech. Sci., 1995, vol. 37, pp. 721-32.

49. X.-L. Gao: Int. J. Solid Struct., 2003, vol. 40, pp. 6445-55.

50. A. Loghman and M.A. Wahab: J. Press. Vess.-Trans. ASME, 1994, vol. 116, pp. 105-09.

51. J. Perry and J. Aboudi: J. Press. Vess.-Trans. ASME, 2003, vol. 125 , pp. $248-52$.

52. G.H. Geiger and D.R. Poirier: Transport Phenomena in Metallurgy, Addison-Wesley Publishing Company, Reading, MA, 1973, p. 396.

53. S. Seghir-Ouali, D. Saury, S. Harmand, O. Phillipart, and D. Laloy: Int. J. Therm. Sci., 2006, vol. 45, pp. 1166-78.

54. S. Harmand, J. Pelle, S. Poncet, and I.V. Shevchuk: Int. J. Therm. Sci., 2013, vol. 67, pp. 1-30.
55. V.R. Voller and C.R. Swaminathan: Num. Heat Transfer B, 1991, vol. 19, pp. 175-89.

56. J. Bohacek, A. Kharicha, A. Ludwig, M. Wu, E. Karimi-Sibaki, A. Paar, M. Brandner, L. Elizondo, and T. Trickl: Appl. Math. Comp., 2017, vol. 319 (C), pp. 301-17.

57. T.F. Coleman and Y. Li: SIAM J. Optimiz., 1996, vol. 6, pp. 418-45.

58. T.F. Coleman and Y. Li: Math. Program., 1994, vol. 67, pp. 189-224.

59. S.J. Orfanidis: Introduction to Signal Processing, Prentice-Hall, Englewood Cliffs, NJ, 1996.

60. R. Brockenbrough and F. Merrit: Structural Steel Designer's Handbook, 5th ed., McGraw-Hill Education, New York, NY, 2011.

61. A. Weronski and T. Hejwowski: Thermal Fatigue of Metals, Marcel Dekker Inc., New York, NY, 1991, p. 223. 